\title{
Thermospermine modulates expression of auxin-related genes in Arabidopsis
}

\author{
Wurina Tong ${ }^{1}$, Kaori Yoshimoto ${ }^{1}$, Jun-Ichi Kakehi ${ }^{1}$, Hiroyasu Motose ${ }^{1}$, Masaru Niitsu ${ }^{2}$ and \\ Taku Takahashi ${ }^{1}$.
}

' Division of Bioscience, Graduate School of Natural Science and Technology, Okayama University, Okayama, Japan

${ }^{2}$ Faculty of Pharmaceutical Sciences, Department of Analytical Chemistry, Josai University, Sakado, Japan

\section{Edited by:}

Ruben Alcazar, Universitat de

Barcelona, Spain

Reviewed by:

Enrico Scarpella, University of

Alberta, Canada

Ruben Alcazar, Universitat de

Barcelona, Spain

Francisco Vera-Sirera, Consejo

Superior de Investigaciones

Cientificas, Spain

\section{*Correspondence:}

Taku Takahashi, Division of

Bioscience, Graduate School of

Natural Science and Technology,

Okayama University, Okayama

700-8530, Japan

e-mail:perfect@cc.okayama-u.ac.jp
Thermospermine, a structural isomer of spermine, is widely distributed in the plant kingdom and has been shown to play a role in repressing xylem differentiation by studies of its deficient mutant, acaulis5 (ac/5), in Arabidopsis. Our results of microarray and realtime PCR analyses revealed that, in addition to a number of genes involved in xylem differentiation, genes related to auxin signaling were up-regulated in acl5 seedlings. These genes include MONOPTEROS, an auxin response factor gene, which acts as a master switch for auxin-dependent procambium formation, and its target genes. Their expression was reduced by exogenous treatment with thermospermine or by transgenic induction of the ACL5 gene. We examined the effect of synthetic polyamines on the expression of these auxin-related genes and on the vascular phenotype of $a c / 5$, and found that tetramines containing the $\mathrm{NC}_{3} \mathrm{NC}_{3} \mathrm{~N}$ chain could mimic the effect of thermospermine but longer polyamines containing the same chain had little or no such effect. We also found that thermospermine had an inhibitory effect on lateral root formation in wild-type seedlings and it was mimicked by synthetic tetramines with the $\mathrm{NC}_{3} \mathrm{NC}_{3} \mathrm{~N}$ chain. These results suggest the importance of the $\mathrm{NC}_{3} \mathrm{NC}_{3} \mathrm{~N}$ chain of thermospermine in its action in modulating auxin signaling.

Keywords: Arabidopsis thaliana, auxin, lateral root, polyamines, thermospermine, xylem

\section{INTRODUCTION}

Polyamines such as spermidine and spermine are abundant in living cells and are involved in a variety of physiological processes including embryogenesis, growth, fruit ripening, and stress responses in plants (Kusano et al., 2007; Alcázar et al., 2010; Takahashi and Kakehi, 2010). Polyamines interact with nucleic acids and membranes and influence many enzymatic reactions. In recent years, attention has been focused on the action of polyamines on the protein synthesis machinery. There is increasing evidence that polyamines stabilize the RNA structure, promote the association of ribosomal subunits, and may affect the rate or efficiency of nascent polypeptide chain elongation (Igarashi and Kashiwagi, 2011). A structural isomer of spermine, thermospermine (Table 1), was first discovered in thermophilic bacteria and has also been implicated in the protein synthesis under extreme environmental conditions (Oshima, 2007). The Arabidopsis acaulis5 (acl5) mutant is defective in the synthesis of thermospermine and shows severe dwarf phenotype, indicating that thermospermine is required for stem elongation (Kakehi et al., 2008). Since ACL5 is predominantly expressed during xylem formation from procambial cells to differentiating xylem vessels (Clay and Nelson, 2005; Muñiz et al., 2008) and acl5 has over-proliferated xylem vessels (Hanzawa et al., 1997), its dwarf phenotype may be primarily attributed to excess xylem differentiation and thermospermine appears to act as a repressor of xylem differentiation. While exogenous thermospermine can partially but significantly rescue the stem growth of acl5, spermine has no such effect (Kakehi et al., 2008). Indeed, acl5 contains spermine produced by the action of spermine synthase, SPMS, while lossof-function mutants of SPMS show wild-type phenotype under normal growth condition (Imai et al., 2004). We have found that norspermine is also able to rescue the growth of acl5 (Kakehi et al., 2010). It is noted that the $\mathrm{NC}_{3} \mathrm{NC}_{3} \mathrm{~N}$ (hereafter expressed as 3-3) arrangement of carbon chains is present in both thermospermine (3-3-4) and norspermine (3-3-3) but not in spermine (3-4-3), suggesting its structural significance for the biological function.

Thermospermine may be widely distributed among the plant kingdom, based on the presence of putative orthologs to ACL5 in different plant species (Knott et al., 2007; Minguet et al., 2008; Takano et al., 2012). Our analyses of suppressor mutants of acl5 named sac have suggested that thermospermine enhances translation of SAC51, a putative basic helix-loop-helix (bHLH) transcription factor gene, by reducing the inhibitory effect of small upstream open reading frames (uORFs) located in its $5^{\prime}$ leader sequence (Imai et al., 2006). A study of the thickvein (tkv) mutant, which represents another allele of the ACL5 locus, suggests that the reduction in polar auxin transport is responsible for the vascular phenotype of the mutant (Clay and Nelson, 2005). Muñiz et al. (2008) revealed that cell death occurs before the onset of secondary cell wall formation in the xylem vessels of acl5, suggesting a role of ACL5 for preventing differentiating xylem vessels from premature programmed cell death (Muñiz et al., 2008). More recently, acl5 mutants were shown to be more sensitive to pathogens than wild-type plants, suggesting a function 
Table 1 | Linear polyamines used in this study.

\begin{tabular}{|c|c|}
\hline Triamines & $\begin{array}{l}\mathrm{H}_{2} \mathrm{~N}\left(\mathrm{CH}_{2}\right)_{a} \mathrm{NH}\left(\mathrm{CH}_{2}\right)_{b} \mathrm{NH}_{2} \\
\text { a-b }\end{array}$ \\
\hline Norspermidine & 3-3 \\
\hline Spermidine & $3-4$ \\
\hline Tetramines & $\begin{array}{l}\mathrm{H}_{2} \mathrm{~N}\left(\mathrm{CH}_{2}\right)_{a} \mathrm{NH}\left(\mathrm{CH}_{2}\right)_{b} \mathrm{NH}\left(\mathrm{CH}_{2}\right)_{c} \mathrm{NH}_{2} \\
\text { a-b-c }\end{array}$ \\
\hline N-(2-Aminoethyl)norspermidine & $3-3-2$ \\
\hline Norspermine & $3-3-3$ \\
\hline Thermospermine & $3-3-4$ \\
\hline N-(5-Aminopentyl)norspermidine & $3-3-5$ \\
\hline Spermine & $3-4-3$ \\
\hline Pentamines & $\begin{array}{l}\mathrm{H}_{2} \mathrm{~N}\left(\mathrm{CH}_{2}\right)_{a} \mathrm{NH}\left(\mathrm{CH}_{2}\right)_{b} \mathrm{NH}\left(\mathrm{CH}_{2}\right)_{c} \\
\mathrm{NH}\left(\mathrm{CH}_{2}\right)_{d} \mathrm{NH}_{2} \\
\text { a-b-c-d }\end{array}$ \\
\hline Homocaldopentamine & $3-3-3-4$ \\
\hline N,N'-Bis(4-aminobutyl)norspermidine & $4-3-3-4$ \\
\hline
\end{tabular}

of thermospermine in defense signaling (Marina et al., 2013). However, the precise mode of action of thermospermine remains to be clarified. For comprehensive understanding of the function of thermospermine in plants, we performed microarray and real-time RT-PCR experiments with acl5 seedlings. Our results revealed that, in addition to a number of genes involved in xylem differentiation, genes related to auxin signaling were up-regulated in acl5 seedlings. They were reduced by thermospermine and synthetic tetramines containing the 3-3 chain (Table 1). These tetramines are further shown to have an inhibitory effect on lateral root formation in wild-type seedlings.

\section{MATERIALS AND METHODS CHEMICALS}

All polyamines used in this study are shown in Table 1. Spermidine, spermine, and norspermidine were purchased from Sigma (St. Louis, MO, USA). The other uncommon polyamines were synthesized by the published method (Niitsu et al., 1992). MS salts for plant nutrition were purchased from Wako (Osaka, Japan).

\section{PLANT MATERIALS AND GROWTH CONDITIONS}

Arabidopsis thaliana accession Columbia-0 (Col-0) was used as the wild type. The original acl5-1 mutant in the accession Landsberg erecta (Ler) was backcrossed more than 7 times into Col-0 (Hanzawa et al., 2000). spms-1 in the Col-0 background has been described previously (Imai et al., 2004). Transgenic acl5-1 lines carrying the HS-ACL5 construct and the SAC51-GUS construct have been described in Hanzawa et al. (2000) and Imai et al. (2006), respectively. In experiments with monopteros $(m p)$ in the Ler background (Berleth and Jürgens, 1993), which were obtained from ABRC, wild-type Ler and acl5-1 in Ler were used as a reference and for crosses. Prior to germination, seeds were surface sterilized with commercial bleach ( $5 \%$ sodium hypochlorite) supplemented with $0.02 \%(\mathrm{v} / \mathrm{v})$ Triton X-100, and rinsed three times with sterile distilled water. For microarray experiments, seeds were germinated and grown on Murashige and Skoog (MS) agar medium supplemented with 3\% sucrose for 7 days under continuous light at $22^{\circ} \mathrm{C}$. For the growth in the presence of polyamines, each polyamine was added to the MS agar or liquid medium at $100 \mu \mathrm{M}$. For treatment of seedlings with polyamines for $24 \mathrm{~h}$ or with heat shock at $37^{\circ} \mathrm{C}$ for $2 \mathrm{~h}$, seeds were germinated, grown in MS solutions for 7 days under continuous light at $22^{\circ} \mathrm{C}$, and incubated as described in figure legends.

\section{RNA EXTRACTION AND REAL-TIME RT-PCR}

Total RNA was isolated from whole seedlings according to the SDS-phenol method and reverse-transcribed with oligo-dT primers and PrimeScript ${ }^{\mathrm{TM}}$ reverse transcriptase (Takara, Kyoto, Japan) at $42^{\circ} \mathrm{C}$ for $1 \mathrm{~h}$. The resulting first-strand cDNA was directly used for real-time PCR with target gene-specific primers (Table 2). PCR reactions were performed using KAPA SYBR FAST qPCR Kit (KAPA Biosystems, Woburn, MA, USA) and the DNA Engine Opticon2 System (Bio-Rad, Hercules, CA, USA). ACTIN8 (At1g49240) was used as an internal standard in the reactions. Data from three independent biological replicates each with two technical replicates are expressed as means $\pm S D$.

\section{MICROARRAY ANALYSIS}

Microarray experiments were performed in three independent biological replicates using the Agilent Arabidopsis 3 oligo microarray (Agilent Technologies, Wilmington, DE, USA) in Hokkaido System Science (Sapporo, Japan). RNA quality was confirmed by gel electrophoresis as well as by OD260/280 nm ratios. The cRNA probes from wild-type and acl5-1 seedlings were labeled with $\mathrm{Cy} 3$ and $\mathrm{Cy} 5$ dyes, respectively, with a Low RNA Fluorescent Linear Amplification Kit (Agilent). After hybridization in Agilent SureHyb chambers, slides were washed according to the manufacturer's instructions and scanned using an Agilent G2505C Microarray Scanner. The scanned images were processed with Feature Extraction software 10.10 (Agilent). The microarray data were submitted to the ArrayExpress database (http://www.ebi. ac.uk/arrayexpress) under accession number E-MTAB-2333. The data were subjected to LOWESS normalization and statistical significance was tested by an unpaired $t$-test with GeneSpring GX software (Agilent). $P<0.05$ were considered to be statistically significant. Gene ontology (GO) analysis was performed using the AgriGO online tool (Zhou et al., 2010) (http://bioinfo.cau.edu. cn/agriGO/analysis.php).

\section{MICROSCOPY}

For observation of xylem development, seedlings were fixed in ethanol/acetic acid (6:1) overnight, incubated twice in $100 \%$ ethanol for $30 \mathrm{~min}$, once in $70 \%$ ethanol for $30 \mathrm{~min}$, and cleared with chloral hydrate:glycerol:water mixture (8:1:2, w:v:v) overnight (Kakehi et al., 2010). Samples were mounted on a microscope slide and examined under differential interference contrast microscopy.

\section{POLYAMINE ANALYSIS}

Polyamines were extracted by grinding $0.5 \mathrm{~g}$ fresh weight of the aerial parts of agar-grown seedlings in liquid nitrogen, 
Table 2 | Primer sequences of the genes used for RT-PCR analysis.

\begin{tabular}{|c|c|c|c|}
\hline Gene name & AGI code & Forward primer sequence & Reverse primer sequence \\
\hline ACL5 & At5g19530 & ACCGTTAACCAGCGATGCTTT & CCGTTAACTCTCTCTTTGATTC \\
\hline ХТН3 & At3g25050 & GTGTTTTTGTAGTAACGTTATGG & GGTTGGATTGAACCAAAGCAA \\
\hline VND7 & At1g71930 & CGATGCATCAATATGGCAAC & AGGGAAGCATCCAAGAGAAT \\
\hline CNA & At1g52150 & GGTATTTGCTGATTCATGAGC & ATGGTTTACACTTGACAGAGC \\
\hline TMO5L1 & At1g68810 & CACCACCAAAACGGATAAAG & CGTTTTGAGACGCATAGCTT \\
\hline PIN1 & At1g73590 & CTTAGCACTGCGGTGATATT & TTGCTGAGCTCCTACTTAAG \\
\hline PIN6 & At1g77110 & CTATCGTACAGGCTGCTCTA & СТССТСAAGAACAACTCTTA \\
\hline YUC2 & At4g13260 & ATGTGGCTAAAGGGAGTGAA & AACTTGCCAAATCGAAACCC \\
\hline IRT1 & At4g19690 & GTCTAATCACTCTAGCCATTGA & TGTATACTCAGCCTGGAGGA \\
\hline
\end{tabular}

suspending the powder in $2.5 \mathrm{ml}$ of $5 \%(\mathrm{w} / \mathrm{v})$ perchloric acid, and incubating on ice for $1 \mathrm{~h}$. After centrifugation at $15,000 \mathrm{~g}$ for $30 \mathrm{~min}, 2 \mathrm{ml}$ of the supernatant was filtered through syringe filter of $0.2 \mu \mathrm{m}$ pore size, neutralized by $1 \mathrm{ml} 2 \mathrm{~N} \mathrm{NaOH}$, incubated with $10 \mu \mathrm{l}$ of benzoyl chloride for $20 \mathrm{~min}$, mixed with $2 \mathrm{ml}$ saturated $\mathrm{NaCl}$ and then added to $2 \mathrm{ml}$ of diethyl ether. After vigorous shaking and centrifugation at $3000 \mathrm{~g}$ for $10 \mathrm{~min}$, the ether layer was evaporated under vacuum and the residue was suspended in $50 \mu \mathrm{l}$ of methanol. The benzoylated sample was analyzed using reverse phase HPLC system equipped with TSKgel ODS-80Ts column (Toso, Tokyo, Japan). The elution was performed with $42 \%$ $(\mathrm{v} / \mathrm{v})$ acetonitrile at a flow rate of $0.5 \mathrm{ml} / \mathrm{min}$ for $50 \mathrm{~min}$ and monitored by measuring its UV absorbance at $254 \mathrm{~nm}$.

\section{RESULTS}

\section{MICROARRAY ANALYSIS OF acL5-1}

To examine the effect of thermospermine deficiency on gene expression profiles in Arabidopsis, we performed microarray experiments with RNA samples from 7-day-old acl51 and wild-type seedlings. The results from three independent experiments identified 173 genes whose transcript level was reproducibly increased more than two-fold in acl5-1 (Supplemental Table 1). GO analysis of these 173 genes revealed significant enrichment of the genes encoding cell wall-related proteins (Supplemental Table 2). These include enzymes involved in cell-wall carbohydrate metabolism such as glycosyl hydrolase family proteins, laccases, pectate lyases, and peroxidases. Representative genes are listed in Table 3. Expression of the genes related to proteolysis such as those encoding serine proteases of the subtilisin family (i.e., SBT1.1 and SBT5.2) and cysteine peptidases (XCP1 and XCP2) were also increased in acl5-1 seedlings. $X C P 1$ has been implicated in the developmental cell death associated with lignified xylem vessel differentiation (Funk et al., 2002). The data also confirmed previous findings that ACL5 and $S A M D C 4 / B U D 2$ are up-regulated in acl5-1, indicating a negative feedback regulation of these polyamine biosynthetic genes by thermospermine (Kakehi et al., 2008). SAMDC4/BUD2 is one of the four genes encoding S-adenosylmethionine decarboxylase, which produces decarboxylated S-adenosylmethionine for the synthesis of higher polyamines (Ge et al., 2006), and has been implicated specifically in the synthesis of thermospermine (Kakehi et al., 2010). With regard to transcription factors, members of the NAC-domain protein gene family (VND1, VND2, VND6, VND7, and XND1), and those of the class III homeodomain leucine-zipper (HD-ZIP III) gene family ( $A T H B 8, P H B$, and $C N A$ ), which are all involved in the regulation of vascular differentiation, were up-regulated in $a c l 5-1$. This is consistent with previous reports that showed up-regulation of the members of these gene families in acl5 mutants (Imai et al., 2006; Kakehi et al., 2008; Muñiz et al., 2008). The mutant also showed increased transcript levels of MYB46, a key player in the regulation of secondary wall biosynthesis in fibers and vessels in stems (Zhong et al., 2007). We further found that the transcript level of MONOPTEROS (MP)/AUXIN RESPONSE FACTOR5 (ARF5), which encodes an auxin-responsive transcription factor (Hardtke and Berleth, 1998), was higher in acl5-1 than in the wild type. In addition, TARGET OF MP 5 (TMO5), which encodes a bHLH transcription factor and has been shown to be a direct target of MP/ARF5 (Schlereth et al., 2010), and TMO5-LIKE1 (TMO5L1) were also up-regulated in acl5-1. As for signaling molecules, PHLOEM INTERCALATED WITH XYLEM/TDIF RECEPTOR (PXY/TDR), which encodes a receptor kinase and acts in the promotion of cambial cell proliferation and in the suppression of its differentiation into xylem (Fisher and Turner, 2007; Hirakawa et al., 2008), was up-regulated in acl5-1.

On the other hand, our results revealed only 14 genes that were down-regulated more than two-fold in acl5-1 (Supplemental Table 3). Only two genes, IRT1, and ZIP8, which are highly homologous to each other and code for a metal transporter (Vert et al., 2002), showed a more than five-fold reduced expression in the mutant.

\section{RESPONSE OF THE SELECTED GENES TO THERMOSPERMINE}

XYLOGLUCAN ENDOTRANSGLYCOSYLASE/HYDROLASE3 (XTH3), VND6, VND7, ATHB8, PXY/TDR, and IRT1 were 
Table 3 | List of representative genes up-regulated in ac/5-1 seedlings.

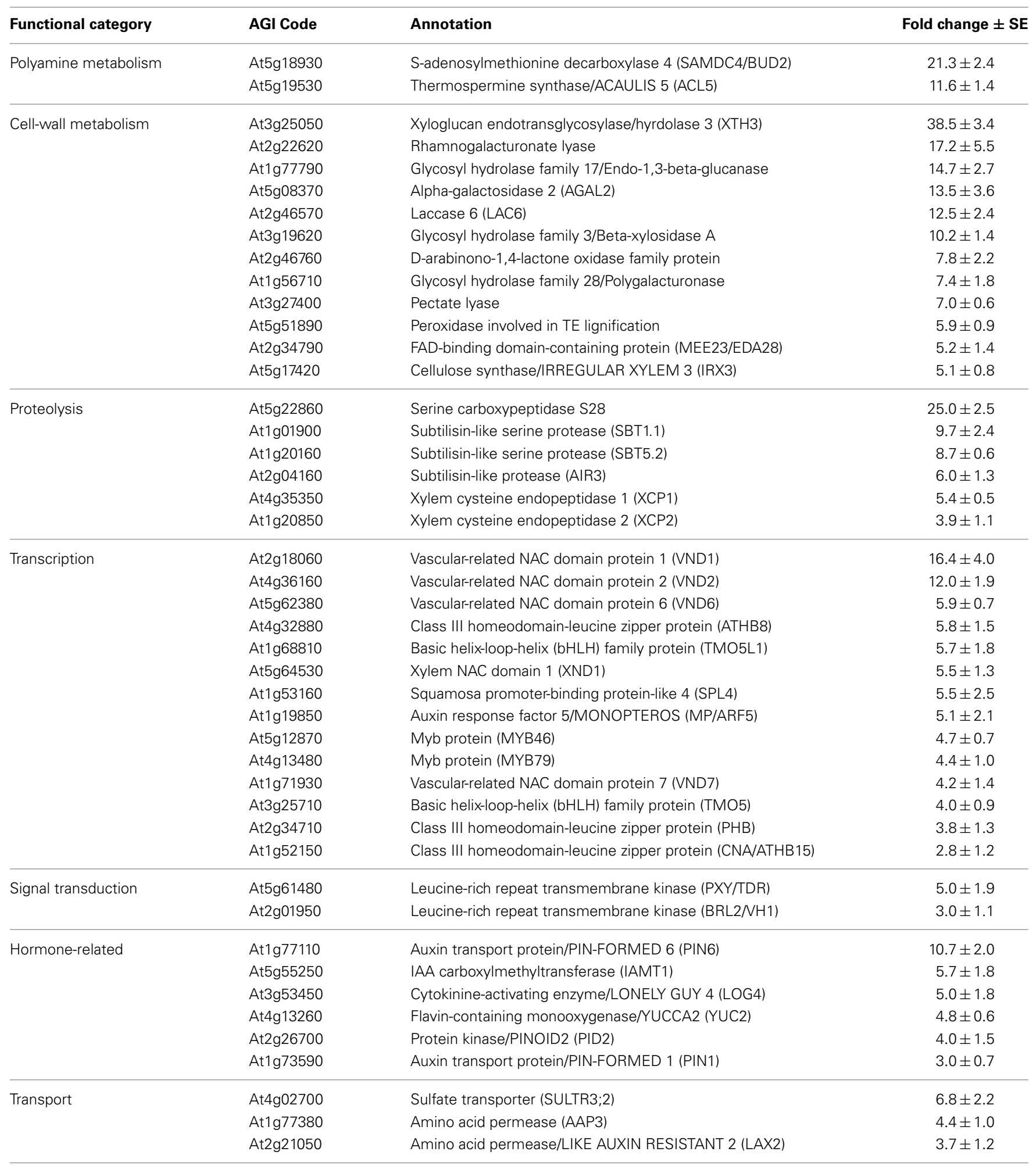

selected for further analysis and their transcript levels were validated by real-time RT-PCR. Our results confirmed that all of these genes except IRT1 were up-regulated in acl5-1 seedlings compared to wild-type seedlings (Figure 1).
We then examined the effect of exogenous application of thermospermine on the expression of these up-regulated genes in acl5-1. Treatment of 7-day-old acl5- 1 seedlings with $100 \mu \mathrm{M}$ thermospermine for $24 \mathrm{~h}$ led to a marked reduction in the transcript 
levels of these genes except for VND7 (Figure 2A). When grown in MS solutions with $100 \mu \mathrm{M}$ thermospermine for 7 days, the acl5-1 seedlings showed a drastic reduction in the transcript levels of all of the genes tested, compared to those grown without thermospermine and those grown with $100 \mu \mathrm{M}$ spermine (Figure 2A). Similar responses of these genes were also observed in wild-type seedlings (Figure 2B).

The effect of thermospermine on gene expression was also examined using transgenic acl5-1 plants carrying a heat-shock inducible ACL5 (HS-ACL5) cDNA. A previous study has shown that the dwarf phenotype of acl5-1 is complemented in a

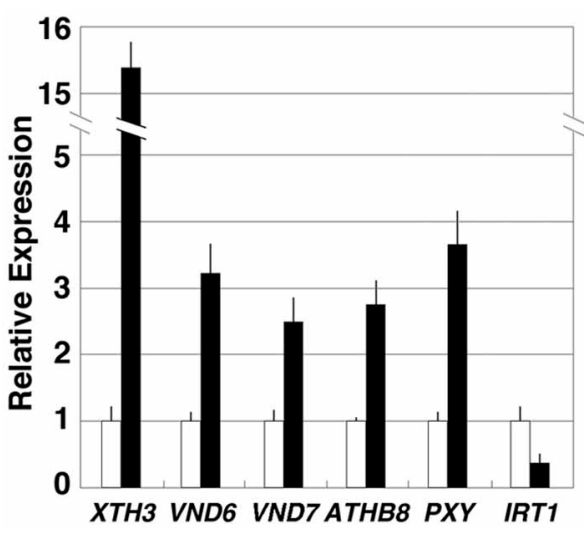

FIGURE 1 | Expression levels of selected genes altered in ac/5-1. Total RNA was prepared from 7-day-old wild-type (Col-0) and ac/5-1 seedlings, and analyzed by quantitative real-time RT-PCR. All transcript levels in ac/5-1 (black bars) are relative to those in the wild type (white bars). ACTIN8 transcripts were used as internal control. Error bars represent the SE $(n=3)$. heat-shock dependent manner by using this transgenic system (Hanzawa et al., 2000). Similar to the case of exogenous treatment for $24 \mathrm{~h}$, transcript levels of all of the genes examined showed a reduction at $24 \mathrm{~h}$ after heat-shock treatment for $2 \mathrm{~h}$ (Figure 2C).

On the other hand, we confirmed that IRT1 expression was reduced in acl5-1 seedlings (Figure 1). However, IRT1 expression was increased neither by treatment with thermospermine in $a c l 5$ 1 and wild-type seedlings nor by heat shock in transgenic acl5-1 plants carrying the HS-ACL5 (not shown).

\section{THERMOSPERMINE NEGATIVELY REGULATES AUXIN-RELATED GENES}

We have recently found that 2,4-D and its derivatives enhance xylem formation in acl5-1 but not in the wild type, suggesting repressive control of auxin-induced xylem formation by thermospermine (Yoshimoto et al., 2012). Auxin-induced procambium formation is mediated by MP/ARF5 (Przemeck et al., 1996; Hardtke and Berleth, 1998), which was listed as an upregulated gene in acl5-1 (Table 3). In addition, genes related to auxin biosynthesis and transport such as PIN-FORMED1 (PIN1), PIN6, and YUCCA2 (YUC2) were also up-regulated in acl5-1 (Table 3). PIN1 and PIN6 encode an auxin efflux carrier (Petrásek et al., 2006), while YUC2 encodes a flavin monooxygenase essential for auxin biosynthesis (Cheng et al., 2006). We thus examined the effect of thermospermine on the expression of these genes by real-time RT-PCR. All of these genes showed higher levels of expression in acl5-1 than in the wild type and were down-regulated by thermospermine in both acl5-1 and wildtype seedlings (Figure 3A). We also confirmed that transcript levels of all of these genes, except for YUC2, were up-regulated by treatment with $100 \mu \mathrm{M} 2,4-\mathrm{D}$ for $24 \mathrm{~h}$ in both wild-type and acl5-1 seedlings (Figure 3B). As reported previously (Hanzawa et al., 2000; Kakehi et al., 2008), ACL5 expression is downregulated by thermospermine (Figure 3A) and up-regulated by
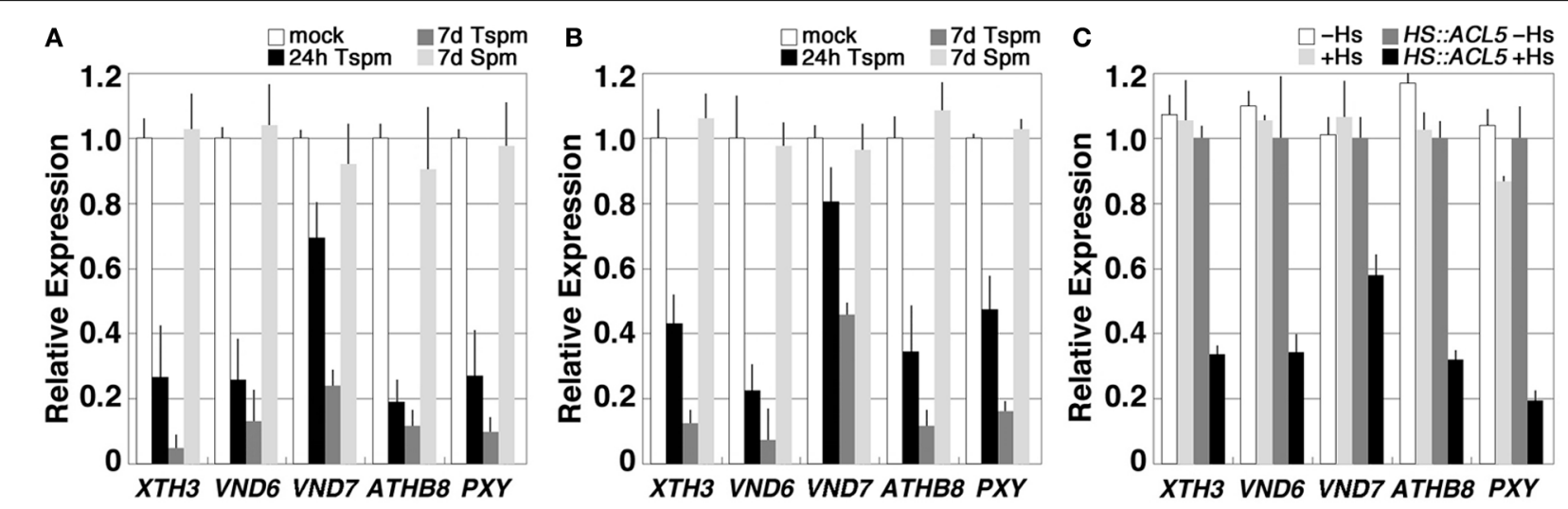

FIGURE 2 | Thermospermine down-regulates the genes that are up-regulated in ac/5-1. (A,B) Effect of exogenous thermospermine and spermine in ac/5-1 (A) and wild-type (B) seedlings on the expression of the genes up-regulated in ac/5-1. Transcript levels in seedlings grown for 7 days in MS solutions and incubated for $24 \mathrm{~h}$ in MS plus $100 \mu \mathrm{M}$ thermospermine (black bars) and those in seedlings grown for 7 days in MS solutions supplemented with thermospermine (dark gray bars) or spermine (light gray bars) are shown relative to those in seedlings grown for 7 days in MS solutions with no polyamines (white bars).
(C) Effect of endogenously-induced thermospermine on the expression of the genes up-regulated in ac/5-1. Transcript levels in ac/5-1 seedlings heat-shocked at $37^{\circ} \mathrm{C}$ for $2 \mathrm{~h}$ followed by 24 - $\mathrm{h}$ culture at $22^{\circ} \mathrm{C}$ (light gray bars), those in transgenic ac/5-1 seedlings carrying the HS-ACL5 construct untreated (dark gray bars) and those in transgenic ac/5-1 seedlings carrying the HS-ACL5 construct heat-shocked at $37^{\circ} \mathrm{C}$ for $2 \mathrm{~h}$ followed by $24-\mathrm{h}$ culture at $22^{\circ} \mathrm{C}$ (black bars), are shown relative to those in ac/5-1 seedlings untreated (white bars). Error bars represent the SE $(n=3)$. 

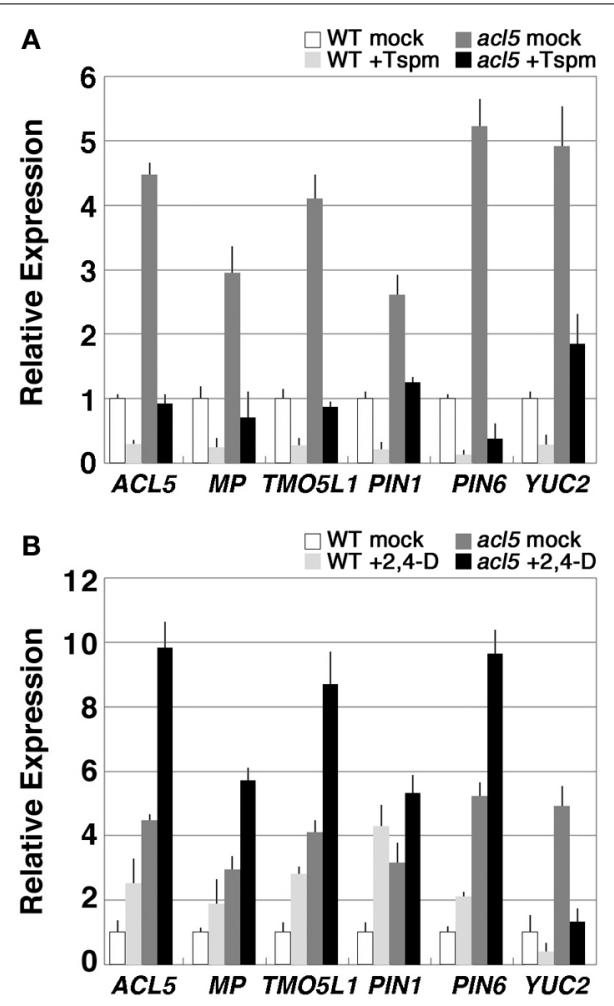

FIGURE 3 | Thermospermine down-regulates the genes that are involved in auxin biosynthesis, transport, and signaling in ac/5-1. (A) Effect of thermospermine on the expression of auxin-related genes. Transcript levels in wild-type seedlings treated for $24 \mathrm{~h}$ with $100 \mu \mathrm{M}$ thermospermine (light gray bars), those in ac/5-1 seedlings treated with mock (dark gray bars) and those in ac/5-1 seedlings treated with thermospermine (black bars), are shown relative to those in wild-type seedlings treated with mock (white bars). (B) Effect of 2,4-D on the expression of auxin-related genes. Transcript levels in wild-type seedlings treated for $24 \mathrm{~h}$ with $100 \mu \mathrm{M} \mathrm{2,4-D}$ (light gray bars), those in ac/5-1 seedlings treated with mock (dark gray bars) and those in ac/5-1 seedlings treated with 2,4-D (black bars), are shown relative to those in wild-type seedlings treated with mock (white bars). Error bars represent the SE $(n=3)$.

2,4-D (Figure 3B). Transcript levels of these genes were also increased in flowering shoots of acl5-1 compared to those of the wild type, and down-regulated by heat shock in transgenic acl5-1 plants carrying the HS-ACL5 (not shown).

We further examined expression of these genes in $m p$, a seedling-lethal allele of MP/ARF5 that lacks basal body structures such as hypocotyl and root meristem (Berleth and Jürgens, 1993). Transcript levels of all of the auxin-related genes examined except for YUC2 were reduced in $m p$ seedlings and were not restored to wild-type levels in $m p$ acl5-1 seedlings (Figure 4), which are morphologically indistinguishable from $m p$.

\section{ARTIFICIAL TETRAMINES CAN MIMIC THE EFFECT OF THERMOSPERMINE}

We next examined the effect of 24-h treatment with synthetic polyamines on ACL5, VND6, and MP/ARF5 in acl5-1 spms-1 seedlings, which produce no tetramines. In addition to thermospermine and norspermine, tetramines of 3-3-2 and 3-3-5

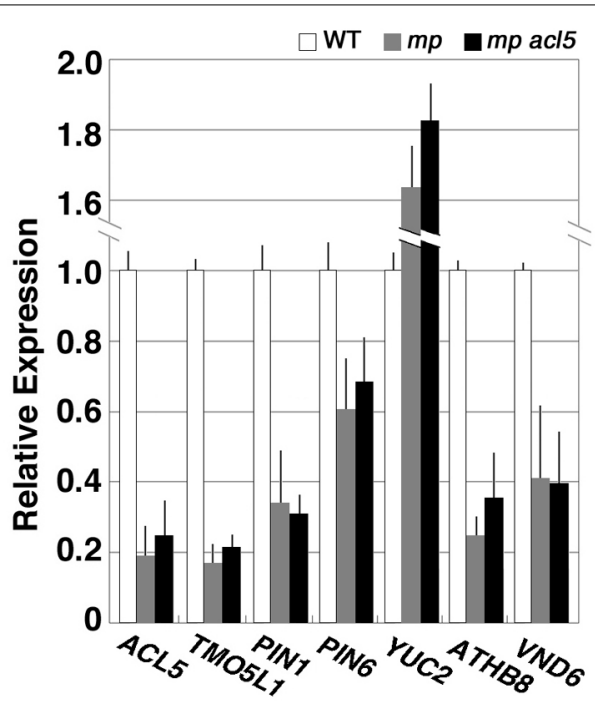

FIGURE 4 | Expression of thermospermine-responsive genes in the $\boldsymbol{m p}$ mutant. Total RNA was prepared from seedlings of the Ler wild type (white bars), mp (gray bars), and mp ac/5-1 (black bars) grown for 7 days in MS agar plates. All transcript levels are relative to those in wild-type seedlings. Error bars represent the SE $(n=3)$.

(Table 1) significantly repressed the expression of these genes, while homocaldopentamine (3-3-3-4) and the 4-3-3-4 pentamine had little or no effect on their expression (Figure 5A). Effect of these synthetic polyamines on xylem development was also examined under microscopy. The acl5-1 spms-1 seedlings were grown in the presence of each polyamine for 7 days. As is the case with thermospermine and norspermine (Kakehi et al., 2010), 3-3-2 and 3-3-5 tetramines drastically reduced excess accumulation of lignin but homocaldopentamine and the 4-3-3-4 pentamine had little or no reduction in acl5-1 spms-1 (Figure 5B). We confirmed that these higher polyamines were absorbed by the root and transported to the shoot by detecting the content of polyamines in the aerial part of the seedlings with HPLC (Figure 5C).

Although our microarray experiments did not identify SAC51 as a gene down-regulated in acl5-1, a previous study revealed that the SAC51 transcript level is increased by thermospermine probably at least in part due to its stabilization (Kakehi et al., 2008). Transgenic acl5-1 seedlings carrying the HS-ACL5 construct described above showed an approximately 2-fold increase in the SAC51 transcript level at $24 \mathrm{~h}$ after heat-shock treatment for $2 \mathrm{~h}$ (Figure 6A). Furthermore, the GUS reporter activity under the control of the SAC51 promoter and its $5^{\prime}$ leader sequence is increased by treatment for $24 \mathrm{~h}$ with thermospermine and norspermine in transgenic acl5-1 seedlings (Kakehi et al., 2010). We therefore examined the continuing effect of synthetic polyamines on SAC51. Transgenic acl5-1 seedlings carrying the GUS reporter gene under the control of the SAC51 promoter and its $5^{\prime}$ leader sequence were grown for 7 days in the presence of each polyamine. The results revealed that the GUS activity was increased by thermospermine, norspermine, 3-3-2, and 3-3-5 tetramines, but unaffected by spermidine, norspermidine, spermine, homocaldopentamine, and the 4-3-3-4 pentamine (Figure 6B). 


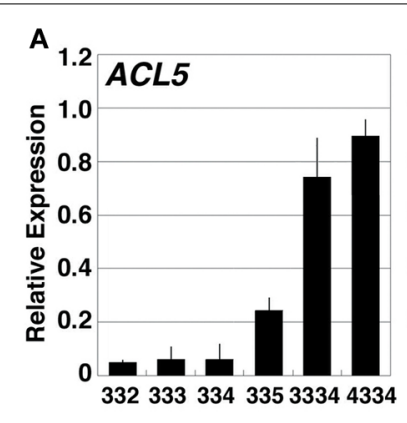

B
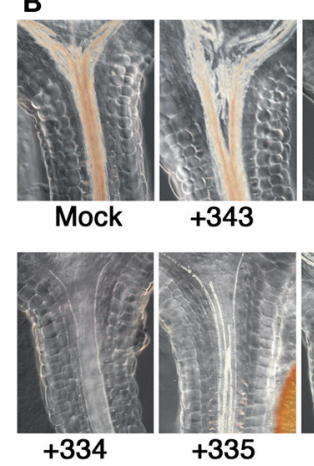

$+334$
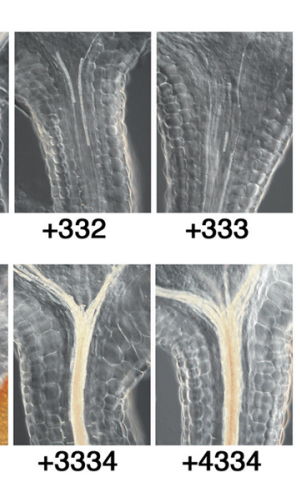

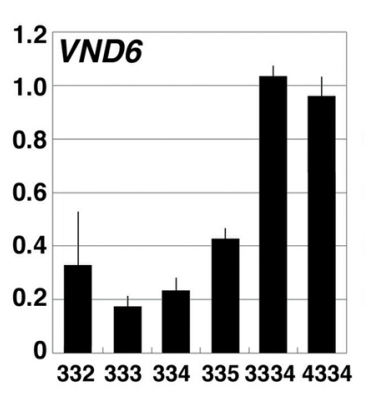

C

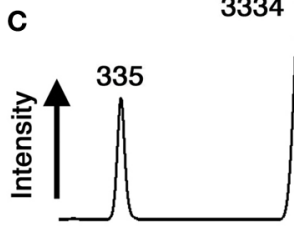

Wild type

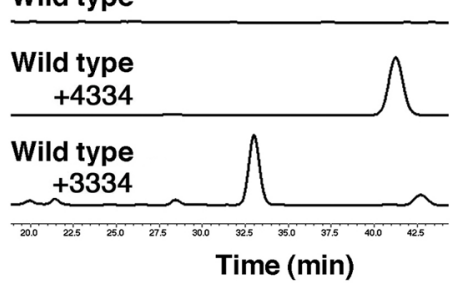

FIGURE 5 | Effect of polyamines in the ac/5-1 spms-1 double mutant. (A) Effect of polyamines on the expression of ACL5, VND6, and MP. Total RNA was prepared from ac/5-1 spms-1 seedlings grown for 7 days in MS solutions and treated for $24 \mathrm{~h}$ in MS plus $100 \mu \mathrm{M}$ each polyamine. Numbers correspond to those of the carbon shown in Table 1. All transcript levels are relative to those in mock-treated ac/5-1 spms-1 seedlings. Error bars represent the SE $(n=3)$. (B) Effect of polyamines on xylem development. acl5-1 spms-1 seedlings were grown for 7 days in MS solutions supplemented with $100 \mu \mathrm{M}$ each polyamine. (C) HPLC separations of benzoylated-polyamines from the aerial part of wild-type seedlings. Seedlings were grown for 7 days in MS agar plates supplemented with homocaldopentamine (C3С3С3C4),

$\mathrm{N}, \mathrm{N}^{\prime}$-bis(4-aminobutyl)norspermidine (C4C3C3C4) or no polyamines. The elution pattern of $\mathrm{C} 3 \mathrm{C} 3 \mathrm{C} 5, \mathrm{C} 3 \mathrm{C} 3 \mathrm{C} 3 \mathrm{C} 4$ and $\mathrm{C} 4 \mathrm{C} 3 \mathrm{C} 3 \mathrm{C} 4$ polyamines is shown above as a reference.

\section{TETRAMINES WITH THE 3-3 CHAIN REPRESS LATERAL ROOT FORMATION}

In the course of the study, we found that exogenous treatment with thermospermine has an inhibitory effect on lateral root formation in both wild-type and acl5-1 seedlings. We then examined whether or not synthetic polyamines have the same effect as thermospermine in wild-type seedlings. The results revealed that lateral root formation was also severely repressed by norspermine, 3-3-2, and 3-3-5 tetramines, but not by spermidine, norspermidine, spermine, homocaldopentamine, and the 4-3-3-4 pentamine (Figure 7).

\section{DISCUSSION}

In bacteria, yeast, and animal cells, effects of polyamines on cell growth were mainly studied at the level of mRNA translation, because polyamines exist to a large extent as RNA-polyamine complexes in cells (Igarashi and Kashiwagi, 2006). A group of genes whose expression is enhanced by polyamines at the level of translation is referred to as a "polyamine modulon." The polyamine modulon includes transcription factors and kinases that in turn activate gene expression of other proteins (Igarashi and Kashiwagi, 2011). In plants, only few genes have been identified as polyamine-responsive genes. In Arabidopsis, spermine up-regulates expression of transcription factor genes such as
WRKY40 and bZIP60. However, since amine oxidase inhibitors cancel this response, it may be triggered by hydrogen peroxide derived from oxidative degradation of spermine (Mitsuya et al., 2009). On the other hand, thermospermine has been shown to negatively regulate expression of ACL5 and the members of the HD-ZIP III gene family such as $A T H B 8$ and PHB (Kakehi et al., 2008). In agreement with the phenotype of acl5-1, which shows excess differentiation of lignified xylem cells, a number of genes involved in the regulation of xylem differentiation and those involved in secondary cell wall formation were up-regulated in acl5-1 seedlings and down-regulated by exogenously supplied and endogenously induced thermospermine. Amongst them was included MP/ARF5. MP/ARF5 encodes a transcription factor of the ARF family, which is activated by auxin-dependent degradation of its interacting repressor, BODENLOS/INDOLE ACETIC ACID-INDUCED PROTEIN 12 (BDL/IAA12), and acts as a master regulator for the establishment of vascular and body patterns in embryonic and post-embryonic development (Berleth and Jürgens, 1993; Przemeck et al., 1996; Hardtke and Berleth, 1998; Mattsson et al., 2003; Hardtke et al., 2004; Weijers et al., 2005). Mutations in MP/ARF5 interfere with the formation of vascular strands and with the initiation of the body axis in the early embryo (Przemeck et al., 1996). In recent studies, such transcription factors as ATHB8 and TMO5 have been identified as a direct 


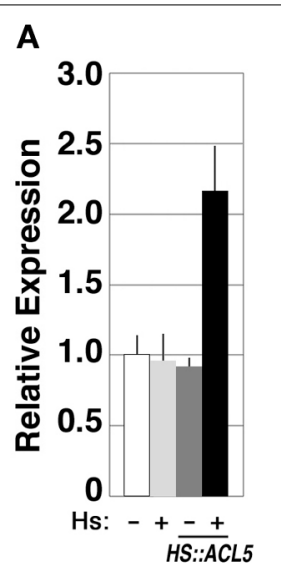

\section{B}

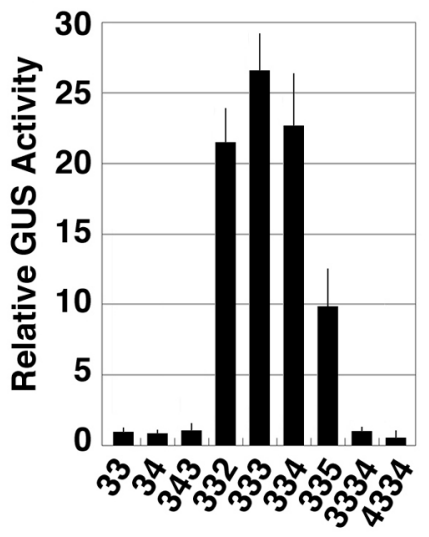

FIGURE 6 | Responses of SAC51 to polyamines in ac/5-1. (A) Effect of endogenously-induced thermospermine on SAC51 expression. Transcript levels in ac/5-1 seedlings heat-shocked at $37^{\circ} \mathrm{C}$ for $2 \mathrm{~h}$ followed by $24-\mathrm{h}$ culture at $22^{\circ} \mathrm{C}$ (light gray bars), those in transgenic ac/5-1 seedlings carrying the HS-ACL5 construct untreated (dark gray bars) and those heat-shocked at $37^{\circ} \mathrm{C}$ for $2 \mathrm{~h}$ followed by 24 -h culture at $22^{\circ} \mathrm{C}$ (black bars), are shown relative to those in ac/5-1 seedlings untreated (white bars). (B) Effect of polyamines on SAC51-GUS expression. Transgenic ac/5-1 seedlings carrying the GUS gene fused to the SAC51 promoter and its 5' leader sequence were grown for 7 days in MS solutions supplemented with each polyamine. Numbers correspond to those of the carbon shown in Table 1. The activity is shown relative to that of mock-treated seedlings. Error bars represent the SE $(n=3)$.
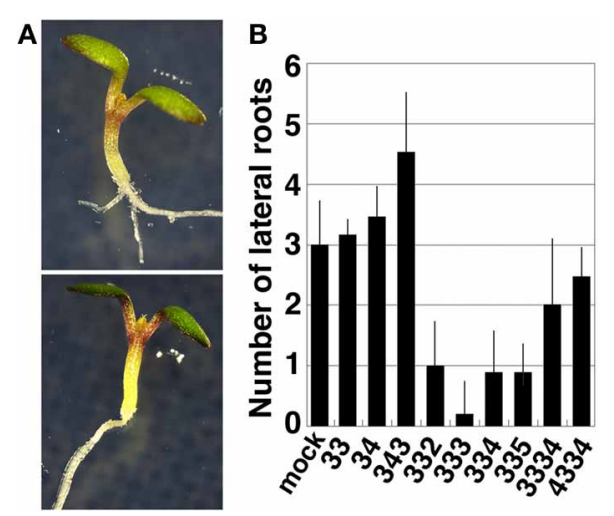

FIGURE 7 | Effect of polyamines on lateral root formation. (A) A wild-type seedling grown for 5 days in MS solutions (upper panel) and that supplemented with $100 \mu \mathrm{M}$ thermospermine (lower panel). (B) Number of lateral roots in wild-type seedlings grown for 5 days in MS solutions supplemented with $100 \mu \mathrm{M}$ each polyamine. Numbers correspond to those of the carbon shown in Table 1. Error bars represent the SE $(n=10)$.

target of MP/ARF5 (Donner et al., 2009; Schlereth et al., 2010). Thus, auxin-induced expression of $A T H B 8$ found in earlier studies (Baima et al., 1995) appears to be mediated by MP/ARF5. $M P / A R F 5$ is also suggested to play a pivotal role in a positive feedback loop for auxin canalization by stimulating expression of PIN1 (Wenzel et al., 2007). We confirmed that transcript levels of these genes were also increased in acl5-1 and decreased by thermospermine. This is possibly due to the effect of MP/ARF5 expression. In fact, they were reduced in $m p$ seedlings. Based on these results together with previous phenotypic analyses of acl5 alleles (Hanzawa et al., 1997; Clay and Nelson, 2005; Imai et al., 2006; Kakehi et al., 2008; Muñiz et al., 2008) and a chemical biology approach revealing that that 2,4-D derivatives enhance the excess xylem phenotype of acl5-1 but have no effect on wild-type xylems (Yoshimoto et al., 2012), it is possible that thermospermine plays an opposing role to auxin in xylem differentiation through negative regulation of the expression of MP/ARF5.

On the other hand, a previous study has shown that knockdown of the HD-ZIP III genes by transgenic overexpression of microRNA miR165, which targets all the five HD-ZIP III genes in Arabidopsis, results in the reduced expression of ACL5 and a subset of the genes related to vascular formation including XCP1, XCP2 and IRX3 (Zhou et al., 2007). These transcript levels were increased along with the acl5-1 mutant transcript level in acl5-1 seedlings (Table 3). Quadruple mutations of the five HD-ZIP III genes cause the lack of metaxylem development and little or no expression of ACL5 in the root tissue (Carlsbecker et al., 2010). These results indicate that the HD-ZIP III transcription factors directly or indirectly activate the ACL5 expression. It is also noted that ACL5 expression was reduced in $m p$ (Figure 4), suggesting that ACL5 expression follows the onset of vascular formation by $M P$. Although the ATHB8 expression is under the direct control of $M P / A R F 5, A T H B 8$ is postulated to play a role in stabilizing procambium precursor cells to narrow regions against perturbations in auxin flow (Donner et al., 2009). Thus, the role of ATHB8 might be virtually performed by thermospermine. In vascular formation, undifferentiated stem cells become procambium precursor cells, procambial cells, xylem precursor cells, and eventually three types of xylem cells: vessels, fibers, and parenchyma cells (Lehesranta et al., 2010). While MP/ARF5 and ATHB8 act in the early steps of this process, downstream thermospermine might participate in a negative feedback loop that fine-tunes $M P$ expression. A previous study suggests that ACL5 functions in preventing xylem precursors from premature cell death before secondary cell wall formation based on the observation that metaxylem vessels and xylem fibers are absent in acl5 mutants (Muñiz et al., 2008). Taking into account the vast effect of thermospermine on the expression of such key regulators as MP/ARF5, HD-ZIP III, and VND genes, it is apparent that thermospermine plays a major inhibitory role against auxin during the process of continuing vascular formation.

Interestingly, we found that exogenous thermospermine strongly represses lateral root formation in wild-type seedlings. Since auxin controls lateral root development through multiple auxin-signaling modules, among which MP/ARF5 is one of major regulators (Lavenus et al., 2013), the inhibitory effect of thermospermine on lateral root formation may also be attributed to the reduced expression of auxin-related genes.

Our microarray study did not identify the regulatory genes whose expression is up-regulated by thermospermine. Given the fact that polyamines have enhancing effects on translation of specific mRNAs, a bHLH transcription factor gene SAC51 whose translation is enhanced by thermospermine is a most probable candidate for a mediator of thermospermine signaling that negatively regulates expression of MP/ARF5 and/or other regulatory 
genes for xylem development. The Arabidopsis genome has three genes homologous to $S A C 51$, all of which contain conserved multiple $\mathrm{uORFs}$ within their long $5^{\prime}$ leader regions. These transcription factors might also be up-regulated at the level of translation by thermospermine. On the other hand, two metal transporter genes, IRT1, and ZIP8, showed reduced expression in acl5-1 mutants. So far, the causal relationship between thermospermine deficiency and the function of these metal transporters remains unknown and needs further investigation.

The results of the experiments using artificial polyamines revealed that, in addition to norspermine, 3-3-2 and 3-35 tetramines are also potent substitutes for thermospermine. Although the efficiency of import and/or transport of polyamines may depend on their structure and size, we confirmed that linear pentamines were absorbed by the root and transported to the shoot but were not able to replace thermospermine in terms of cellular functions. Thus, we suggest that the tetramines containing the core structure of 3-3 are biologically active and they may be useful as novel plant growth regulators against auxin for the control of xylem differentiation and lateral root formation. Since ACL5 orthologs are present in unicellular algaes (Knott et al., 2007), the original role of thermospermine may be unrelated to cell differentiation in multicellular organisms. It is of interest to speculate that, while retaining the mode of action at the molecular level, thermospermine may have been recruited as a part of a negative feedback control in auxin-induced proliferation of xylem cells, which are programmed to die, during the evolution of vascular systems in land plants. Future work should be done with non-vascular plants to address the original function of thermospermine.

\section{ACKNOWLEDGMENTS}

This work was supported in part by the Japan Society for the Promotion of Science (JSPS) Grant-in-Aid for Scientific Research No. 22370021 to Taku Takahashi and that on Innovative Areas No. 25119715 to Hiroyasu Motose, respectively.

\section{SUPPLEMENTARY MATERIAL}

The Supplementary Material for this article can be found online at: http://www.frontiersin.org/journal/10.3389/fpls. 2014.00094/abstract

Supplemental Table 1 | List of the genes up-regulated in ac/5-1 seedlings.

Supplemental Table 2 | GO analysis of the gene up-regulated in ac/5-1 seedlings.

Supplemental Table 3 | List of the genes down-regulated in ac/5-1 seedlings.

\section{REFERENCES}

Alcázar, R., Altabella, T., Marco, F., Bortolotti, C., Reymond, M., Koncz, C., et al. (2010). Polyamines: molecules with regulatory functions in plant abiotic stress tolerance. Planta 231, 1237-1249. doi: 10.1007/s00425-010-1130-0

Baima, S., Nobili, F., Sessa, G., Lucchetti, S., Ruberti, I., and Morelli, G. (1995). The expression of the Athb- 8 homeobox gene is restricted to provascular cells in Arabidopsis thaliana. Development 121, 4171-4182.

Berleth, T., and Jürgens, G. (1993). The role of the MONOPTEROS gene in organising the basal body region of the Arabidopsis embryo. Development 118, $575-587$.
Carlsbecker, A., Lee, J. Y., Roberts, C. J., Dettmer, J., Lehesranta, S., Zhou, J., et al. (2010). Cell signalling by microRNA165/6 directs gene dose-dependent root cell fate. Nature 465, 316-321. doi: 10.1038/nature08977

Cheng, Y., Dai, X., and Zhao, Y. (2006). Auxin biosynthesis by the YUCCA flavin monooxygenases controls the formation of floral organs and vascular tissues in Arabidopsis. Genes Dev. 20, 1790-1799. doi: 10.1101/gad.1415106

Clay, N. K., and Nelson, T. (2005). Arabidopsis thickvein mutation affects vein thickness and organ vascularization, and resides in a provascular cell-specific spermine synthase involved in vein definition and in polar auxin transport. Plant Physiol. 138, 767-777. doi: 10.1104/pp.104.055756

Donner, T. J., Sherr, I., and Scarpella, E. (2009). Regulation of preprocambial cell state acquisition by auxin signaling in Arabidopsis leaves. Development 136, 3235-3246. doi: 10.1242/dev.037028

Fisher, K., and Turner, S. (2007). PXY, a receptor-like kinase essential for maintaining polarity during plant vascular-tissue development. Curr. Biol. 17, 1061-1066. doi: 10.1016/j.cub.2007.05.049

Funk, V., Kositsup, B., Zhao, C., and Beers, E. P. (2002). The Arabidopsis xylem peptidase XCP1 is a tracheary element vacuolar protein that may be a papain ortholog. Plant Physiol. 128, 84-94. doi: 10.1104/pp.010514

Ge, C., Cui, X., Wang, Y., Hu, Y., Fu, Z., Zhang, D., et al. (2006). BUD2, encoding an S-adenosylmethionine decarboxylase, is required for Arabidopsis growth and development. Cell Res. 16, 446-456. doi: 10.1038/sj.cr.7310056

Hanzawa, Y., Takahashi, T., and Komeda, Y. (1997). ACL5: an Arabidopsis gene required for internodal elongation after flowering. Plant J. 12, 863-874. doi: 10.1046/j.1365-313X.1997.12040863.x

Hanzawa, Y., Takahashi, T., Michael, A. J., Burtin, D., Long, D., Pineiro, M., et al. (2000). ACAULIS5, an Arabidopsis gene required for stem elongation, encodes a spermine synthase. EMBO J. 19, 4248-4256. doi: 10.1093/emboj/19. 16.4248

Hardtke, C. S., and Berleth, T. (1998). The Arabidopsis gene MONOPTEROS encodes a transcription factor mediating embryo axis formation and vascular development. EMBO J. 17, 1405-1411. doi: 10.1093/emboj/17.5.1405

Hardtke, C. S., Ckurshumova, W., Vidaurre, D. P., Singh, S. A., Stamatiou, G., Tiwari, S. B., et al. (2004). Overlapping and non-redundant functions of the Arabidopsis auxin response factors MONOPTEROS and NONPHOTOTROPIC HYPOCOTYL 4. Development 131, 1089-1100. doi: 10.1242/dev.00925

Hirakawa, Y., Shinohara, H., Kondo, Y., Inoue, A., Nakanomyo, I., Ogawa, M., et al. (2008). Non-cell-autonomous control of vascular stem cell fate by a CLE peptide/receptor system. Proc. Natl Acad. Sci. U.S.A. 105, 15208-15213. doi: 10.1073/pnas.0808444105

Igarashi, K., and Kashiwagi, K. (2006). Polyamine modulon in Escherichia coli: genes involved in the stimulation of cell growth by polyamines. J. Biochem. 139, 11-16. doi: 10.1093/jb/mvj020

Igarashi, K., and Kashiwagi, K. (2011). Characterization of genes for polyamine modulon. Methods Mol. Biol. 720, 51-65. doi: 10.1007/978-1-61779-034-8_3

Imai, A., Akiyama, T., Kato, T., Sato, S., Tabata, S., Yamamoto, K. T., et al. (2004). Spermine is not essential for survival of Arabidopsis. FEBS Lett. 556, 148-152. doi: 10.1016/S0014-5793(03)01395-4

Imai, A., Hanzawa, Y., Komura, M., Yamamoto, K. T., Komeda, Y., and Takahashi, T. (2006). The dwarf phenotype of the Arabidopsis acl5 mutant is suppressed by a mutation in an upstream ORF of a bHLH gene. Development 133, 3575-3585. doi: 10.1242/dev.02535

Kakehi, J. I., Kuwashiro, Y., Motose, H., Igarashi, K., and Takahashi, T. (2010). Norspermine substitutes for thermospermine in the control of stem elongation in Arabidopsis thaliana. FEBS Lett. 584, 3042-3046. doi: 10.1016/j.febslet.2010.05.035

Kakehi, J. I., Kuwashiro, Y., Niitsu, Y., and Takahashi, T. (2008). Thermospermine is required for stem elongation in Arabidopsis thaliana. Plant Cell Physiol. 49, 1342-1349. doi: 10.1093/pcp/pcn 109

Knott, J. M., Römer, P., and Sumper, M. (2007). Putative spermine synthases from Thalassiosira pseudonana and Arabidopsis thaliana synthesize thermospermine rather than spermine. FEBS Lett. 581, 3081-3086. doi: 10.1016/j.febslet.2007.05.074

Kusano, T., Yamaguchi, K., Berberich, T., and Takahashi, Y. (2007). Advances in polyamine research in 2007. J. Plant Res. 120, 345-350. doi: 10.1007/s10265007-0074-3

Lavenus, J., Goh, T., Roberts, I., Guyomarc'h, S., Lucas, M., De Smet, I., et al. (2013). Lateral root development in Arabidopsis: fifty shades of auxin. Trends Plant Sci. 18, 450-458. doi: 10.1016/j.tplants.2013.04.006 
Lehesranta, S. J., Lichtenberger, R., and Helariutta, Y. (2010). Cell-to-cell communication in vascular morphogenesis. Curr. Opin. Plant Biol. 13, 59-65. doi: 10.1016/j.pbi.2009.09.004

Marina, M., Sirera, F. V., Rambla, J. L., Gonzalez, M. E., Blázquez, M. A., Carbonell, J., et al. (2013). Thermospermine catabolism increases Arabidopsis thaliana resistance to Pseudomonas viridiflava. J. Exp. Bot. 64, 1393-1402. doi: 10.1093/jxb/ert012

Mattsson, J., Ckurshumova, W., and Berleth, T. (2003). Auxin signaling in Arabidopsis leaf vascular development. Plant Physiol. 131, 1327-1339. doi: 10.1104/pp.013623

Minguet, E. G., Vera-Sirera, F., Marina, A., Carbonell, J., and Blázquez, M. A. (2008). Evolutionary diversification in polyamine biosynthesis. Mol. Biol. Evol. 25, 2119-2128. doi: 10.1093/molbev/msn161

Mitsuya, Y., Takahashi, Y., Berberich, T., Miyazaki, A., Matsumura, H., Takahashi, H., et al. (2009). Spermine signaling plays a significant role in the defense response of Arabidopsis thaliana to cucumber mosaic virus. J. Plant Physiol. 166, 626-643. doi: 10.1016/j.jplph.2008.08.006

Muñiz, L., Minguet, E. G., Singh, S. K., Pesquet, E., Vera-Sirera, F., MoreauCourtois, C. L., et al. (2008). ACAULIS5 controls Arabidopsis xylem specification through the prevention of premature cell death. Development 135, 2573-2582. doi: 10.1242/dev.019349

Niitsu, M., Sano, H., and Samejima, K. (1992). Syntheses of tertiary tetraamines and quaternary pentaamines with three and four methylene chain units. Chem. Pharm. Bull. 40, 2958-2961. doi: 10.1248/cpb.40.2958

Oshima, T. (2007). Unique polyamines produced by an extreme thermophile, Thermus thermophilus. Amino Acids 33, 367-372. doi: 10.1007/s00726-0070526-z

Petrásek, J., Mravec, J., Bouchard, R., Blakeslee, J. J., Abas, M., Seifertová, D., et al. (2006). PIN proteins perform a rate-limiting function in cellular auxin efflux. Science 312, 914-918. doi: 10.1126/science.1123542

Przemeck, G. K., Mattsson, J., Hardtke, C. S., Sung, Z. R., and Berleth, T. (1996). Studies on the role of the Arabidopsis gene MONOPTEROS in vascular development and plant cell axialization. Planta 200, 229-237. doi: 10.1007/BF0 0208313

Schlereth, A., Möller, B., Liu, W., Kientz, M., Flipse, J., Rademacher, E. H., et al. (2010). MONOPTEROS controls embryonic root initiation by regulating a mobile transcription factor. Nature 464, 913-916. doi: 10.1038/ nature 08836

Takahashi, T., and Kakehi, J. I. (2010). Polyamines: ubiquitous polycations with unique roles in growth and stress responses. Ann. Bot. 105, 1-6. doi: 10.1093/aob/mcp259

Takano, A., Kakehi, J. I., and Takahashi, T. (2012). Thermospermine is not a minor polyamine in the plant kingdom. Plant Cell Physiol. 53, 606-616. doi: $10.1093 / \mathrm{pcp} / \mathrm{pcs} 019$
Vert, G., Grotz, N., Dédaldéchamp, F., Gaymard, F., Guerinot, M. L., Briat, J. F., et al. (2002). IRT1, an Arabidopsis transporter essential for iron uptake from the soil and for plant growth. Plant Cell 14, 1223-1233. doi: 10.1105/tpc. 001388

Weijers, D., Benkova, E., Jäger, K. E., Schlereth, A., Hamann, T., Kientz, M., et al. (2005). Developmental specificity of auxin response by pairs of ARF and Aux/IAA transcriptional regulators. EMBO J. 24, 1874-1885. doi: 10.1038/sj.emboj.7600659

Wenzel, C. L., Schuetz, M., Yu, Q., and Mattsson, J. (2007). Dynamics of MONOPTEROS and PIN-FORMED1 expression during leaf vein pattern formation in Arabidopsis thaliana. Plant J. 49, 387-398. doi: 10.1111/j.1365313X.2006.02977.x

Yoshimoto, K., Noutoshi, Y., Hayashi, K., Shirasu, K., Takahashi, T., and Motose, H. (2012). A chemical biology approach reveals an opposite action between thermospermine and auxin in xylem development in Arabidopsis thaliana. Plant Cell Physiol. 53, 635-645. doi: 10.1093/pcp/pcs017

Zhong, R., Richardson, E. A., and Ye, Z. H. (2007). The MYB46 transcription factor is a direct target of SND1 and regulates secondary wall biosynthesis in Arabidopsis. Plant Cell 19, 2776-2792. doi: 10.1105/tpc.107.053678

Zhou, D., Xin, Z., Yi, L., Zhenhai, Z., and Zhen, S. (2010). agriGO: a GO analysis toolkit for the agricultural community. Nucleic Acids Res. 38, W64-W70. doi: 10.1093/nar/gkq310

Zhou, G. K., Kubo, M., Zhong, R., Demura, T., and Ye, Z. H. (2007). Overexpression of miR165 affects apical meristem formation, organ polarity establishment and vascular development in Arabidopsis. Plant Cell Physiol. 48, 391-404. doi: $10.1093 / \mathrm{pcp} / \mathrm{pcm} 008$

Conflict of Interest Statement: The authors declare that the research was conducted in the absence of any commercial or financial relationships that could be construed as a potential conflict of interest.

Received: 10 January 2014; accepted: 25 February 2014; published online: 14 March 2014.

Citation: Tong W, Yoshimoto K, Kakehi J-I, Motose H, Niitsu M and Takahashi T (2014) Thermospermine modulates expression of auxin-related genes in Arabidopsis. Front. Plant Sci. 5:94. doi: 10.3389/fpls.2014.00094

This article was submitted to Plant Metabolism and Chemodiversity, a section of the journal Frontiers in Plant Science.

Copyright (C) 2014 Tong, Yoshimoto, Kakehi, Motose, Niitsu and Takahashi. This is an open-access article distributed under the terms of the Creative Commons Attribution License (CC BY). The use, distribution or reproduction in other forums is permitted, provided the original author(s) or licensor are credited and that the original publication in this journal is cited, in accordance with accepted academic practice. No use, distribution or reproduction is permitted which does not comply with these terms. 